\title{
Downregulation of FLNa and RUNx3 Expression are Involved in the Progression of Colorectal Adenocarcinoma Disease
}

\author{
${ }^{1}$ Qi-Lian Liang, ${ }^{1}$ Yuan Zhou, ${ }^{2}$ Zhou-Yu Li, ${ }^{1}$ Qiu-Long Liu and ${ }^{1}$ Guo-Qiang Chen \\ ${ }^{1}$ Department of Oncology, Affiliated Hospital of Guangdong Medical College, \\ 524001 Zhanjiang, China \\ ${ }^{2}$ Department of Radiotherapy, Affiliated Tumor Hospital of Guangzhou Medical College, \\ 510095 Guangzhou, China
}

\begin{abstract}
To study the expressions of Filamin A (FLNa) and RUNx3 in human Colorectal Adenocarcinoma (CRA) tissues and explore their correlation with the initiation and progression of colorectal adenocarcinoma. The expression levels of FLNa and RUNx3 protein in the samples which came from 80 patients of CRA tissues and adjacent normal colorectal tissues ( $10 \mathrm{~cm}$ distance away from the margin of CRA) were examined by using immunohistochemical Streptavidin-Peroxidase (S-P) Method, Western-blotting and Reverse Transcription Polymerase Chain Reaction (RT-PCR). The correlation between their expression and the clinic pathologic features was analyzed. The positive rates of FLNa and RUNx 3 in CRA tissues were 52.5\% (42/80) and 46.3\% (37/80), respectively while in adjacent normal colorectal tissues they were $87.5 \%(70 / 80)$ and $91.3 \%(73 / 80)$, respectively. The difference was significant $(\mathrm{p}<0.0000)$. Expression of FLNa was related positively with expression of RUNx3 in CRA tissues ( $r=0.358, p=0.0002)$. Expression levels of FLNa and RUNx3 were correlated with the TNM staging, lymph node metastasis and the depth of tumor invasion but not with the patient's age, gender lesion site and tumor size. The expression of RUNx3 has nothing to do with the degree of tumor differentiation but FLNa was opposite. The results of RT-PCR and Western blot were consistent with that of immunohistochemistry. FLNa and RUNx3 will be important factors in predicting colorectal adenocarcinoma biological behavior and judging prognosis and it also may be new target $\mathrm{s}$ for colorectal adenocarcinoma targeted therapy.
\end{abstract}

Key words: Colorectal adenocarcinoma, filam in $\mathrm{A}, \mathrm{RUNx} 3$, Western blotting, immunohistochemistry, reverse transcriptase polymerase chain reaction

\section{INTRODUCTION}

Colorectal adenocarcinoma is one of the most frequent malignancies of the gastrointestinal tract in China and the morbidity of it has increased year by year. The prognosis of CRA can be influenced by tumor recrudescence and distant metastasis. Filamin A belongs to Actin-binding proteins, served as an important scaffold protein in signal transduction and it is involved with a large number of signal transduction pathways that related to tumor formation (Balduini, 2011; Kolahi and Mofrad, 2008), FLNa plays crucial role in the occurrence and development of CRA. RUNx 3 is a new recently discovered tumor suppressor gene. The tumor-suppressor mechanism of RUNx3 is closely related to TGF- $\beta$ pathway, a signal transduction pathway that involved in growth inhibition and apoptosis inducing (Tong et al., 2009; Bae and Choi, 2004; Ito and Miyazono, 2003). In malignant tumors, RUNx3 is frequently inactivated by heterozygote deletion and promoter region hypermethylation (Homma et al., 2006). Both of FLNa and RUNx3 have close relationships with signal transduction pathways that related to tumor formation and it is not clear whether these two factors have synergistic effects. Researchers chose them for study as FLNa and RUNx3 may be important factors in predicting CRA biological behavior. In this study, the expression of FLNa and RUNx3 in CRA tissues and adjacent normal colorectal tissues were examined by using immunohistochemical Streptavidin-Peroxidase (S-P) Method and then RT-PCR and Western blotting were further used to detect the expression of FLNa and RUNx3 in the level of transcription and protein.

\section{MATERIALS AND METHODS}

Samples material: About of 80 patients with CRA tissues who had unabridged case file were collected from the Department of Surgery in Affiliated Hospital of

Corresponding Author: Qi-Lian Liang, Department of Oncology, Affiliated Hospital of Guangdong Medicine College 57, People Avenue, 524001 Zhanjiang, Guangdong, China 
Table 1: The primers of FLNa, RUNx 3 and GAPDH

\begin{tabular}{lll}
\hline Primers & Upstream primer sequence & Downstream primer sequence \\
\hline FLNa & 5'-AGCCTCCACGAGACATCATC-3' & 5'-CCAGTCTGTACTCCCCCTTG-3' \\
RUNx3 & 5'-ATCCCCTGACAAAAAGTGACG-3' & 5'-GCAAAGCAGTTCCACCCA-3' \\
GAPDH & 5'-GAAGGTGAAGGTCGGAGTC-3' & 5'-GAAGATGGTGATGGGATTTC-3 \\
\hline
\end{tabular}

Guangdong Medical College. The adenocarcinoma tissue was from the central of the tumor. The control group was normal tissues which was parted from CRA tissues about $10 \mathrm{~cm}$. Among these cases, 49 were male and 31 were female. The range of age was $44-78$ years with a median age of 62. All of them were finally diagnosed and classified by histopathology. None of the patients received chemotherapy or radiotherapy before surgical therapy. Complete medical and pathological records were available.

Main reagent: Immunohistochemistry $\mathrm{S}-\mathrm{P}$ kit was purchased from Zhongshan Jinqiao Bio-Tech Ltd., Beijing; DAB developer was purchased from Wuhan Boster Bio-Tech Ltd., Wuhan; Trizol kit and Radio Immunpoprecipitation Assay (RIPA) were purchased from Fuzhou Maixin Bio-Tech Ltd., Fuzhou, China; PCR primer was purchased from Shanghai Shenggong Bio-Tech Ltd., Shanghai, China; rabbit anti-human FLNa monoclonal antibody and rabbit anti-human RUNx3 monoclonal antibody were purchased from Santa cruz Bio-Tech Ltd., USA.

Immunohistochemistry detection and assay: Paraffinembedded blocks were cut into $4 \mu \mathrm{m}$ serial sections, immunohistochemical staining was carried out by the standard S-P technique and concrete procedure was conducted according to the S-P kit specification. Using the human uterus coloration tissue as the positive control of FLNa while the breast cancer tissue with RUNx3 positive expressed was used as the positive control of RUNx3. PBS-replaced primary antibody as the negative control. Result judgment criteria: five visions were chosen by light microscope to observe and count the cell then calculated the average cell-positive rate according to stained, extent and distribution of cancer cells. Specific criteria were as follows: divided the immunohistochemical staining results into three degree. Positive cells $<25 \%$, light stained and no obvious particles were defined as negative (-); positive cells $>25$ and $<50 \%$, stained yellow, sparse granules were seen as weak positive $(+)$; positive cells $>50 \%$, brown staining, diffuse particles were defined as strong positive $(++)$.

Western blotting: The tissue was homogenized by using RIPA lysis buffer and then the total protein was extracted and quantitated from CRA tissues as well as adjacent normal colorectal tissues. The protein was subjected to
SDS-PAGE gel electrophoresis. After transferring the protein to nitrocellulose membranes, blocked the membranes with TBST which contain $5 \%$ skimmed milk for $1 \mathrm{~h}$ at room temperature. Then, the membranes were labeled with primary antibody (rabbit anti-human RUNx3 monoclonal antibody, rabbit anti-human FLNa monoclonal antibody and anti-GAPDH antibody) overnight at $-4^{\circ} \mathrm{C}$ next day, the membranes were washed with TBST for three times, each time for $10 \mathrm{~min}$. And then added the secondary antibody for $1 \mathrm{~h}$ at $37^{\circ} \mathrm{C}$. The expression of protein band was visualized by using chemiluminescence.

RNA extraction and RT-PCR assay: The total RNA was extracted from CRA tissues and adjacent normal colorectal tissues by using Trizol kit. The concentration of total RNA was assayed by spectrophotometry. Two micrograms of total RNA was reverse transcribed to obtain cDNA by using reverse transcriptase. GAPDH served as the internal reference. The PCR primers were designed according to the cDNA of FLNa, RUNx3 and GAPDH in the GenBank. The primers of FLNa, RUNx3 and GAPDH were shown in Table 1. And cDNA was amplified and synthesized by PCR. The PCR conditions for FLNa and RUNx 3 were 35 cycles of $94^{\circ} \mathrm{C}(5 \mathrm{~min}), 94^{\circ} \mathrm{C}(30 \mathrm{sec})$ $55^{\circ} \mathrm{C}(30 \mathrm{sec})$ and $72^{\circ} \mathrm{C}(30 \mathrm{sec})$. The final extension was at $72^{\circ} \mathrm{C}$ for $5 \mathrm{~min}$ then placed at $4^{\circ} \mathrm{C}$. The internal reference was carried out in the same system and the condition was similar to FLNa and RUNx3. The produces of RT-PCR were separated by agarose gel electrophoresis, scanned by ultraviolet photometry.

Statistical analysis: All statistical analyses were performed using the SPSS Software package. The data were analyzed by the $\chi^{2}$-test, Spearman Coefficient Correlation test and t-test. Values of $\mathrm{p}<0.05$ were considered statistically significant.

\section{RESULTS AND DISCUSSION}

The expression of FLNa and RUNx3 detected by immunohistochemical Streptavidin-Peroxidase (S-P) Method (Table 2): The positive expression of FLNa was located in cytoplasm and the positive expression of RUNx3 was located in cytoplasm and nucleus (Fig. 1). The positive rates of FLNa and RUNx3 in CRA tissues were $52.5 \%(42 / 80)$ and $6.3 \%(37 / 80)$, respectively while in adjacent normal colorectal tissues they were $87.5 \%(70 / 80)$ and $91.3 \%(73 / 80)$, respectively. The difference was 

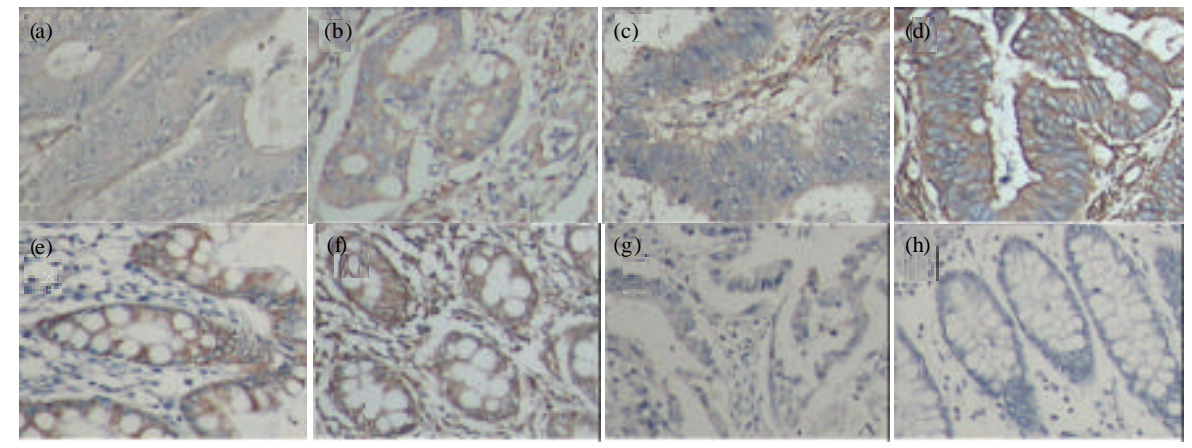

Fig. 1: Expression of RUNx3 and FLNa in colorectal adenocarcinoma tissues and adjacent colorectal adenocarcinoma tissues, detected by immunohistochemistry (sp, 200); a) week positive expression of RUNx3 in colorectal adenocarcinoma tissues; b) week positive expression of FLNa in colorectal adenocarcinoma tissues; c) strong positive expression of RUNx3 in colorectal adenocarcinoma tissues; d) positive expression of FLNa in colorectal adenocarcinoma tissues; e) positive expression of RUNx3 in colorectal adenocarcinoma tissues; f) positive expression of FLNa in adjacent colorectal adenocarcinoma tissues; g) negative expression in colorectal adenocarcinoma tissues; $h$ ) negative expression in adjacent colorectal adenocarcinoma tissues

Table 2: Expressions of FLNa and RUNx3 in normal colorectal tissues and colorectal adenocarcinoma tissues detected by immunohistochemistry

\begin{tabular}{lllcccc}
\hline & & FLNa & & RUNx3 & & \\
Groups & $\mathrm{N}$ & Positive expression & $\chi^{2}$ & p-value & Positive expression & $\chi^{2}$ \\
Normal tissues & 80 & $70(87.50)$ & 23.34 & 0.000 & $73(91.25)$ & 18.85 \\
Adenocarcinoma tissues & 80 & $42(52.50)$ & - & - & $37(46.25)$ & - \\
\hline
\end{tabular}

Table 3: Relationship between FLNa and RUNx3 expressions in colorectal adenocarcinoma tissues $(\mathrm{N}=80, \mathrm{n})$

\begin{tabular}{lrrrll}
\hline & 38 FLNa & & & \\
RUNX3 & \multicolumn{1}{c}{-} & & & & \\
\hline-43 & 30 & 5 & 8 & 0.3581 & 0.0002 \\
+ & 2 & 11 & 7 & - & - \\
++ & 6 & 5 & 6 & - & - \\
\hline
\end{tabular}

significant $(\mathrm{p}<0.0000)$ in addition, the expression of FLNa and RUNx3 is hierarchical so researchers use the Spearman nonparametric correlation analysis to analyze the experimental data. The result of Spearman test showed that Expression of FLNa was related weak positively with expression of RUNx 3 in CRA tissues $(r=0.358, p=0.0002$, Table 3).

Relationship between the clinicopathological characteristics and the expressions of FLNa and RUNx3 (Table 4): The result of immunohistochemical was analyzed by statistics. The expressions of FLNa and RUNx 3 were associated to depth of invasion, TNM stage and lymph node metastasis $(\mathrm{p}<0.05)$ but not with the patient's age, gender and tumor size $(p>0.05)$. The expression of RUNx3 was related to Differentiation on the contrary the expression of FLNa was not.
The expression of FLNa and RUNx3 mRNAs detected by RT-PCR: GAPDH served as the internal reference and researchers used the value of $\mathrm{FLNa} / \mathrm{GAPDH}$ and RUNx3/GAPDH for quantitative in RT-PCR. The results showed that the expression of FLNa mRNA in carcinoma tissues was lower than that in the normal tissues $[(0.20 \pm 0.04)$ vs. $(0.92 \pm 0.03)$, $\mathrm{p}<0.05]$ while the expression of RUNx 3 mRNA in carcinoma tissues was also lower than that in the normal tissues $[(0.34 \pm 0.05)$ vs. $(0.59 \pm 0.06)$, $\mathrm{p}<0.05$ ] (Table 5). The difference was statistically valid.

The expression of FLNa and RUNx 3 proteins detected by Western blotting: GAPDH served as the internal reference and researchers used the value of FLNa/GAPDH and RUNx3/GAPDH for quantitative in Western blotting. The results showed that the expression of FLNa protein in carcinoma tissues was lower than that in the normal tissues $[(0.17 \pm 0.04)$ vs. $(0.78 \pm 0.06), \mathrm{p}<0.05]$ while the expression of RUNx3 protein in carcinoma tissues was also lower than that in the normal tissues $[(0.41 \pm 0.05)$ vs. $(0.57 \pm 0.06), \mathrm{p}<0.05]$ (Table 6). The difference was statistically valid. 
J. Anim. Vet. Adv., 11 (18): 3411-3416, 2012

Table 4: Relationship between the expressions of FLNa and RUNx3 in colorectal adenocarcinoma tissues and the clinicopathological characteristics

\begin{tabular}{|c|c|c|c|c|c|c|c|}
\hline \multirow[b]{2}{*}{ Characteristics } & \multirow[b]{2}{*}{$\mathrm{N}$} & \multicolumn{3}{|l|}{ FLNa } & \multicolumn{3}{|l|}{ RUNx3 } \\
\hline & & Positive & $\chi^{2}$ & $\mathrm{p}$-value & Positive & $\chi^{2}$ & p-value \\
\hline \multicolumn{8}{|l|}{ Age(year) } \\
\hline$\geq 60$ & 54 & $26(48.15)$ & 1.2618 & 0.2613 & $24(44.44)$ & 0.2179 & 0.6407 \\
\hline$<60$ & 26 & $16(61.54)$ & - & - & $13(50.00)$ & - & - \\
\hline \multicolumn{8}{|l|}{ Gender } \\
\hline Male & 49 & $23(46.94)$ & 1.5682 & 0.2105 & $21(42.86)$ & 0.5856 & 0.4441 \\
\hline Female & 31 & $19(61.29)$ & - & - & $16(51.61)$ & - & - \\
\hline \multicolumn{8}{|l|}{ Tumorsize (cm) } \\
\hline$\geq 5$ & 37 & $16(43.24)$ & 2.3653 & 0.1241 & $20(54.05)$ & 1.6865 & 0.1941 \\
\hline$<5$ & 43 & $26(60.47)$ & - & - & $17(39.53)$ & - & - \\
\hline \multicolumn{8}{|l|}{ Differentiation } \\
\hline Well & 19 & $11(57.89)$ & 2.4550 & 0.2950 & $11(57.89)$ & 7.6478 & 0.0218 \\
\hline Moderately & 37 & $16(43.24)$ & - & - & $11(29.73)$ & - & - \\
\hline poorly & 24 & $15(62.50)$ & - & - & $15(62.50)$ & - & - \\
\hline \multicolumn{8}{|c|}{ Lymph node metastasis } \\
\hline No & 49 & $31(63.27)$ & 5.8766 & 0.0153 & $27(57.14)$ & 4.3542 & 0.0369 \\
\hline Yes & 31 & $11(35.48)$ & - & - & $10(32.26)$ & - & - \\
\hline \multicolumn{8}{|c|}{ Depth of invasion } \\
\hline Not to serosa & 47 & $33(70.21)$ & 14.3349 & 0.0002 & $27(57.45)$ & 5.7461 & 0.0165 \\
\hline To serosa & 33 & $9(27.27)$ & - & - & $10(30.30)$ & - & - \\
\hline \multicolumn{8}{|l|}{ TNM staging } \\
\hline $\mathrm{I}+\mathrm{II}$ & 44 & $31(70.45)$ & 12.6397 & 0.0004 & $27(61.36)$ & 8.9844 & 0.0027 \\
\hline$\underline{\mathrm{III}}+\mathrm{IV}$ & 36 & $11(30.56)$ & - & - & $10(27.78)$ & - & - \\
\hline
\end{tabular}

Table 5: The expression of FLNa and RUNx3 mRNAs detected by RT-PCR

\begin{tabular}{|c|c|c|c|c|c|c|}
\hline \multirow[b]{2}{*}{ Expression } & \multicolumn{3}{|l|}{ FLNa } & \multicolumn{3}{|l|}{ RUNx3 } \\
\hline & Positive rate $(\%)$ & $\mathrm{FLNa} / \mathrm{GAPDH}$ & $p$-value & Positive rate (\%) & RUNx3/GAPDH & $p$-value \\
\hline $\begin{array}{l}\text { Normal tissues } \\
\text { Adenocarcinoma tissues }\end{array}$ & $\begin{array}{l}68(85 \%) \\
39(48.75 \%)\end{array}$ & $\begin{array}{l}0.92 \pm 0.03 \\
0.20 \pm 0.04\end{array}$ & $\begin{array}{l}<0.01 \\
-\end{array}$ & $\begin{array}{l}71(88.75 \%) \\
38(47.5 \%)\end{array}$ & $\begin{array}{l}0.59 \pm 0.06 \\
0.34 \pm 0.05\end{array}$ & $\begin{array}{l}<0.01 \\
-\end{array}$ \\
\hline
\end{tabular}

Table 6: The expression of FLNa and RUNx3 proteins detected by RT-PCR

\begin{tabular}{|c|c|c|c|c|c|c|}
\hline \multirow[b]{2}{*}{ Expression } & \multicolumn{3}{|l|}{ FLNa } & \multicolumn{3}{|l|}{ RUNx3 } \\
\hline & Positive rate (\%) & FLNa/GAPDH & $\mathrm{p}$-value & Positive rate (\%) & RUNx3/GAPDH & $p$-value \\
\hline Normal tissues & $65(81.25 \%)$ & $0.78 \pm 0.06$ & $<0.01$ & $68(85 \%)$ & $0.57 \pm 0.06$ & $<0.01$ \\
\hline Adenocarcinoma tissues & $35(43.75 \%)$ & $0.17 \pm 0.04$ & $40(50 \%)$ & $0.41 \pm 0.05$ & - & - \\
\hline
\end{tabular}

\section{CONCLUSION}

FLNa is an important scaffold protein in signal transduction pathway. FLNa dimeric subunits have an Actin Binding Domains (ABDs) and 24 repeat $\beta$-pleated sheet units. They are separated by two hinges sequences. $\mathrm{V}$-shaped homodimer that is related to FLNa's function is formed by two polypeptide chains (Nakamura et al., 2007). FLNa mainly locate in cytoplasm. The FLNa $\beta$-pleated sheet units formed by repeated sequence provide interface for protein-protein interaction. So that it can interact with many proteins which have important functions (Robertson et al., 2003). FLNa combining with actin can influence the cytoskeleton remodeling and cell movement so, it plays an important role in migration and invasion behavior of tumor cell (Yamazaki et al., 2005). Earlier studies find that FLNa also combines with numerous protein other than actin including epidermal growth factor receptor (Fiori et al., 2009), Caveolinreceptor (Ravid et al., 2008), Prion Protein (Li et al., 2009), P73a protein (Kim et al., 2007), Carcinoembryonic antigen-related cell adhesion molecule (Klaile et al., 2005).
FLNa can impact the expression of a variety of cell receptors together with other actin-binding proteins in cell (Keshamouni et al., 2006) by means of the MAPK/ERK pathway (He et al., 2003), PI3K/AKt pathway (Ravid et al., 2008), TGF- $\beta$ signaling pathway (Sasaki et al., 2001) to inhibit migration and invasion of tumor cell. Bedolla et al. (2009) detected the expression of FLNa protein in prostate cancer tissue specimens by using immunohistochemical, discovered that the expression of FLNa in Metastatic prostate cancer was significantly reduced in the cytoplasm while it was significantly increased in the nucleus compared with benign prostate and prostatic intraepithelial neoplasia and carcinoma in situ. Maybe this phenomenon is related to increased degree of malignancy of cancer cells. The current study shows that the expression of FLNa was significantly reduced in malignant tumors compared with normal tissue. The In-depth study of FLNa's role in the mechanism of the tumor development and progression can provide new ideas for cancer diagnosis and treatment.

RUNx3 is an important transcription as well as a member of RUNX tumor suppressor gene family. Human 
RUNx3 gene is located on chromosome $1 \mathrm{p} 36.1$ and is a heterodimer composed of $\alpha$ and $\beta$ subunits (Bangsow et al., 2001; Coffman, 2003; Bagchi and Mills, 2008). The abnormal expression of FLNa is an important tumor prognostic factor and the low expression of FLNa has a close relationship with promoter methylation and heterozygote deletion (Tong et al., 2009; Homma et al., 2006). RUNx3's lower expression can imbalance proliferation and differentiation of gastrointestinal epithelial, behaved as gastrointestinal epithelial cell hyperplasia or abnormal differentiation and thus participates in the development and progression of gastrointestinal tumors; it also can increase the infiltration and metastasis of tumor cells. Manish $M$ found that RUNx3 inactivation due to promoter hypermethylation in colorectal polyps represents an early event in colorectal cancer progression (Subramaniam et al., 2009). Methylation RUNx3 can re-expression after demethylation. RUNx3 re-expression can inhibit tumor growth and induce apoptosis of tumor cells. At present, most studies suggest that RUNx3's tumor suppressor mechanism is closely related to TGF- $\beta$ pathway which has the function of growth inhibition and apoptosis inducing (Tong et al., 2009; Bae and Choi, 2004; Ito and Miyazono, 2003). Chromosome 1 p36.1 also contains a number of other tumor suppressor genes such as RIZ1, CHD5, PLA2G2A and TP73, etc., these tumor suppressor genes and RUNx3 may collaborate with each other to co-induced tumors (Bagchi and Mills, 2008). Imamura et al. (2005) detected 92 cases of colorectal cancer by using MSP in which 31/92 (34\%) tumors could detected RUNx3 methylation and poorly differentiated tumor and other differentiated tumor were significant different in histologically $(\mathrm{p}=0.028)$ in these tumors with methylation. RUNx3 methylation plays an important role in colorectal cancer, especially in poorly differentiated tumors. $\mathrm{Ku}$ et al. (2004) alsoobtained similar results by using RT-PCR.

This experimental result showed that the positive expression rates of FLNa and RUNx3 in colorectal adenocarcinoma were lower than that in normal colorectal tissues. The expression of FLNa and RUNx3 had no relationship with age, gender and tumor size and TNM staging but were correlated with lymph node metastasis and the depth of tumor invasion. RUNx3 was correlated with the degree of tumor differentiation while the FLNa was not. These two factors had low positive correlation. The results of RT-PCR and Western blot were consistent with that of immunohistochemistry.

Though both of them can influence tumor cell proliferation and migration by several signal pathways, it's not clear whether FLNa and RUNx3 had a synergistic effect in tumor development. So, next step researchers can further study at that phenomenon.

Since, FLNa is a critical scaffold protein in cell signaling pathway, involved in the regulation of cell proliferation, adhesion, migration and other cell behavior and $R U N \times 3$ is an important tumor suppressor gene in tumors, its re-expression can inhibit tumor growth and induce tumor apoptosis. The expression of FLNa and RUNx3 is closely connected with biological behavior of colorectal cancer so the downregulation of FLNa and RUNx3 expression can be considered as indicators to judge the degree of colorectal cancer invasion in the further research they also can be expected as a target for the control and treatment of colorectal adenocarcinoma invasion and metastasis.

\section{ACKNOWLEDGEMENT}

Researchers appreciate the help from Dr. Han-Guo Jiang, Department of Pathology, Guangdong Medical College. Lian Liang and Yuan Zhou contributed equally to this research.

\section{REFERENCES}

Bae, S.C. and J.K. Choi, 2004. Tumor suppressor activity of RUNX3. Oncogene, 23: 4336-4340.

Bagchi, A. and A.A. Mills, 2008. The quest for the $1 \mathrm{p} 36$ tumor suppressor. Cancer Res., 68: 2551-2556.

Balduini, A., 2011. New aspects of filamin a versatility. Blood, 118: 2029-2030.

Bangsow, C., N. Rubins, G. Glusman, Y. Bernstein and V. Negreanu et al., 2001. The RUNX3 genesequence, structure and regulated expression. Gene, 279: $221-232$.

Bedolla, R.G., Y. Wang, A. Asuncion, K. Chamie and S. Siddiqui et al., 2009. Nuclear versus cytoplasmic localization of filamin a in prostate cancer: Immunohistochemical correlation with metastases. Clin. Cancer Res., 15: 788-796.

Coffman, J.A., 2003. Runx transcription factors and the developmental balance between cell proliferation and differentiation. Cell. Biol. Int., 27: 31 5-324.

Fiori, J.L., T.N. Zhu, M.P. O'Connell, K.S. Hoek and F.E. Indig et al., 2009. Filamin a modulates kinase activation and intracellular trafficking of epidermal growth factor receptors in human melanoma cells. Endocrinology, 150: 2551-2560.

He, H.J., S. Kole, Y.K. Kwon, M.T. Crow and M. Bernier, 2003. Interaction of filamin a with the insulin receptor alters insulin-dependent activation of the mitogen-activated protein kinase pathway. J. Biol. Chem., 278: 27096-27104. 
Homma, N., G. Tamura, T. Honda, Y. Matsumoto, S. Nishizuka, S. Kawata and T. Motoyama, 2006. Spreading of methylation within RUNX3 CpG island in gastric cancer. Cancer Sci., 97: 51-56.

Imamura, Y., K. Hibi, M. Koike, M. Fujiwara, Y. Kodera, $\mathrm{K}$. Ito and A. Nakao, 2005. RUNX3 promoter region is specifically methylated in poorly-differentiated colorectal cancer. Anticancer Res., 25: 2627-2630.

Ito, Y. and K. Miyazono, 2003. RUNX transcription factors as key targets of TGF- $\beta$ superfamily signaling. Curr. Opin. Genet. Dev., 13: 43-47.

Keshamouni, V.G., G. Michailidis, C.S. Grasso, S. Anthwal and J.R. Strahler et al., 2006. Differential protein expression profiling by iTRAQ-2DLC-MS/MS of lung cancer cells undergoing epithelial-mesenchymal transition reveals a migratory/invasive phenotype. J. Proteome Res., 5: 1143-1154.

Kim, E.J., J.S. Park and S.J. Um, 2007. Filamin a negatively regulates the transcriptional activity of $\mathrm{p} 73 \alpha$ in the cytoplasm. Biochem. Biophys. Res. Commun., 362: 1101-1106.

Klaile, E., M.M. Muller, C. Kannicht, B.B. Singer and L. Lucka, 2005. CEACAM1 functionally interacts with filamin A and exerts a dual role in the regulation of cell migration. J. Cell. Sci., 118: 5513-5524.

Kolahi, K.S. and M.R.K. Mofrad, 2008. Molecular mechanics of filamin's rod domain. Biophys J., 94: 1075-1083.

Ku, J.L., S.B. Kang, Y.K. Shin, H.C. Kang and S.H. Hong et al., 2004. Promoter hypermethylation downregulates RUNX3 gene expression in colorectal cancer cell lines. Oncogene, 23: 6736-6742.
Li, C., S. Yu, F. Nakamura, S. Yin and J. Xu et al., 2009. Binding of pro-prion to filamin A disrupts cytoskeleton and correlates with poor prognosis in pancreatic cancer. J. Clin. Invest., 119: 2725-2736.

Nakamura, F., T.M. Osbiorn, C.A. Hartemink, J.H. Hartwig and T.P. Stossel, 2007. Structure basis of filamin a functions. J. Cell. Biol., 179: 1011-1025.

Ravid, D., D. Chuderland, L. Landsman, Y. Lavie, R. Reich and M. Liscovitch, 2008. Filamin a is a novel caveolin-1-dependent target in IGF-I-stimulated cancer cell migration. Exp. Cell. Res., 314: 2762-2773.

Robertson, S.P., S.R.F. Twigg, A.J. Sutherland-Smith, V. Biancalana and R.J. Gorlin et al., 2003. Localized mutations in the gene encoding the cytoskeletal protein filamin A cause diverse malformations in humans. Nat. Genet., 33: 487-491.

Sasaki, A., Y. Masuda, Y. Ohta, K. Ikeda and K. Watanabe, 2001. Filamin associates with Smads and regulates transforming growth factor-beta signaling. J. Biol. Chem., 276: 17871-17877.

Subramaniam, M.M., J.Y. Chan, R. Soong, K. Ito and K.G. Yeoh et al., 2009. RUNX3 inactivation in colorectal polyps arising through different pathways of colonic carcinogenesis. Am. J. Gastroenterol., 104: 426-436.

Tong, D.D., Y. Jiang, M. Li, D. Kong and X.N. Meng et al., 2009. RUNX3 inhibits cell proliferation and induces apoptosis by TGF- $\beta$ dependent and-independent mechanisms in human colon carcinoma cells. Pathobiology, 76: 163-169.

Yamazaki, D., S. Kurisu and T. Takenawa, 2005. Regulation of cancer cell motility through actin reorganization. Cancer Sci., 96: 379-386. 